Annales Geophysicae (2002) 20: 349-364 (C) European Geophysical Society 2002

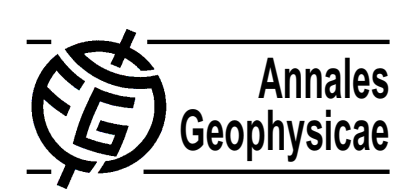

\title{
Two types of ion spectral gaps in the quiet inner magnetosphere: Interball-2 observations and modeling
}

\author{
N. Y. Buzulukova ${ }^{1}$, Y. I. Galperin ${ }^{1,(\dagger)}$, R. A. Kovrazhkin ${ }^{1}$, A. L. Glazunov ${ }^{1}$, G. A. Vladimirova ${ }^{1}$, H. Stenuit ${ }^{2}$, \\ J. A. Sauvaud ${ }^{2}$, and D. C. Delcourt ${ }^{3}$ \\ ${ }^{1}$ IKI, Moscow, Russia \\ ${ }^{2} \mathrm{CESR} / \mathrm{CNRS}$, Toulouse, France \\ ${ }^{3}$ CETP - CNRS, Saint-Maur des Fosses, France \\ ${ }^{(\dagger)}$ passed away on the 28 December 2001
}

Received: 26 February 2001 - Revised: 17 August 2001 - Accepted: 10 September 2001

\begin{abstract}
We analyse measurements of ion spectral gaps (ISGs) observed by the ION particle spectrometer on board the Interball-2 satellite. The ISG represents a sharp decrease in $\mathrm{H}^{+}$flux at a particular narrow energy range. ISGs are practically always observed in the inner magnetosphere in a wide MLT range during quiet times. Clear examples of ISG in the morning, dayside, evening and nightside sectors of the magnetosphere are selected for detailed analysis and modeling. To obtain a model ISG, the trajectories of ions drifting in the equatorial plane from their nightside source to the observation point were computed for the energy range $0.1-15 \mathrm{keV}$. Three global convection models (McIlwain, 1972, 1986; Volland, 1973; Stern, 1975) were tested to reproduce the observed ISGs in all MLT sectors. Qualitative agreement is obtained for all three models, but the better agreement for quiet times is reached with the McIlwain (1972) convection model. It is shown that the ISGs observed by the ION spectrometer throughout the inner magnetosphere are the result of superposition of the two effects, already described in the literature (e.g. McIlwain, 1972; Shirai et al., 1997), but acting under different conditions. Also, the role of particle source location on the model gaps is investigated. It may be concluded that despite the evidence of large amplitude and directional local fluctuations of electric fields in the inner magnetosphere (Quinn et al., 1999), the existence of a stationary average convection pattern is confirmed by this modeling. This fact directly follows from observations of ISGs and from a good agreement of observations with modeled gaps calculated in the frames of adiabatic theory for a stationary (average) convection pattern.
\end{abstract}

Key words. Magnetospheric physics (plasma convection;

Correspondence to: N. Buzulukova (nat@aster.iki.rssi.ru) electric fields)

\section{Introduction}

A characteristic feature of proton spectra often observed by the ION experiment on board Interball-2 in the inner magnetosphere (L-shells from $\sim 5$ to $\sim 10$ in a wide MLT range) is a sharp decrease in the $\mathrm{H}^{+}$flux in specific energy ranges (see Sauvaud et al., 1998b,a). This phenomenon is referred to as ion spectral gap (ISG), and the energy which corresponds to the gap center is refered to as the gap energy. ISGs were first observed by McIlwain (1972) on board the geostationary satellite ATS-5 during relatively quiet times. In these early observations, the gap energy decreased from $10 \mathrm{keV}$ to $1 \mathrm{keV}$ as MLT was changing from 0 to $16 \mathrm{~h}$. The existence of ISGs was explained by McIlwain as the result of enhanced proton drift time from the source to the observation point at geostationary orbit, i.e. ISG forms when the ion drift time at a particular energy is comparable to or larger than the magnetospheric lifetime of this ion. In the adiabatic approximation, protons undergo $\boldsymbol{E} \times \boldsymbol{B}$ drift (independently of energy and charge) and magnetic gradient/curvature drift (energy and charge dependent). During their motion across L-shells, these ions experience adiabatic (betatron and Fermi-type) energization. In the inner nightside magnetosphere, the corotation component of the $\boldsymbol{E} \times \boldsymbol{B}$ drift velocity points eastward, whereas the gradient drift velocity of protons points westward, so that the drift time at particular "resonant" energies increases dramatically (up to $\sim 100 \mathrm{~h}$ according to McIlwain, 1972). This time is larger than the proton lifetime expected from wave-particle interactions and charge exchange losses. 
The proton flux at such "resonant" energies will accordingly decrease, yielding ISG in energy - time spectrograms.

McIlwain (1972) constructed an empirical model of the convection electric field for quiet times by tracing proton trajectories backward in time from the observation point at geostationary orbit to some "injection boundary" in the magnetotail which was viewed as the ion source. Mcllwain used an empirical relationship between the ISG energy and local time for fitting the convection model to the observed shape of the particle spectra. According to the "injection boundary" model, particles injected (or energized) during short substorm intervals $(\sim 5 \mathrm{~min}$ which is considerably smaller than the characteristic time of steady convection), fill the region of the magnetosphere tailward of some empirically defined sharp boundary (DeForest and McIlwain, 1971). Subsequently, particles drift in the steady convection field given by the model. Mcllwain thus defined simultaneously the convection model and the model particle source. Plasma injection was viewed as a short burst followed by a long steady drift. This approach proved fruitful and the convection models thus constructed allowed one to describe in detail the particle (both ions and electrons) spectra recorded by ATS-5. These concepts were developed further in several subsequent studies of the inner magnetosphere (see, for example, Mauk and Meng, 1983; Greenspan et al., 1985).

Besides the "injection boundary" for the description of the particle injection process into the inner magnetosphere, other models have been published such as:

(a) energized by a wave propagating from the tail to the inner magnetosphere (Moore et al., 1981);

(b) innermost limit of substorm-related magnetic field changes and particle injection resulting from disruption of the cross-tail current sheet (Lopez et al., 1990);

(c) narrow MLT channels of enhanced electric field in the nightside magnetosphere (probably related to the dipolarization process) along which particles rapidly penetrate down to low L-shells (Li et al., 1998);

(d) continuous flux of particles from the magnetotail energized in the course of nonadiabatic scattering (Delcourt et al., 1996).

Some of these concepts are equivalent, or at least, not mutually exclusive, while some are alternative. This illustrates that up until now there is no clear understanding of the physical processes at the origin of particle injections into the inner magnetosphere. It is still unclear whether the low-energy particle flow in the inner magnetosphere follows from largescale convection from the tail in the global steady convection field or it is the net result of a multitude of small injections during localized substorm-like events, or both. The other side of this problem is to what extent the "injection boundary" model can be used during "quiet times".

Clearly, the agreement between observed ISGs and model calculations of ion drifts will be very sensitive to the magnetic and electric field models assumed. While the magnetic field on low L-shells where ISGs are observed (i.e. typically from $\mathrm{L} \sim 5$ up to $\mathrm{L} \sim 10$ ) may be considered as fairly well known with minor deviations from one model to the other, global convection models in this region vary considerably. For example, in the McIlwain (1986) convection model, equipotentials in the evening sector are open for $K_{p}=0$ and $\mathrm{L} \approx 7$ (i.e. they connect the tail source with the magnetopause on the dayside), whereas in McIlwain (1972), these same equipotentials are closed. The structure of the ion gaps calculated from these models will differ accordingly.

Kovrazhkin et al. (1999) tested the assumption of enhanced drift times at "resonant" energies as being responsible for ISGs in Interball-2 measurements. Using the VollandStern convection model (Volland, 1973; Stern, 1975), the model ion gaps were calculated in the $\sim 4$ to $\sim 20 \mathrm{~h}$ MLT range and a dependence of the ISGs energy upon MLT similar to that of McIlwain was obtained. On the other hand, Shirai et al. (1997) also performed drift path calculations using the Volland-Stern convection model and concluded that the ISGs observed by the AKEBONO satellite in the morning sector follow from the formation of "forbidden zones" that are inaccessible to the ions launched from the magnetotail. The aim of this work is to test and compare three widely used convection models:

(a) Mcllwain (1972);

(b) McIlwain (1986);

(c) Volland-Stern (Volland, 1973; Stern, 1975)

within ranges of MLT and L-shells that are as wide as possible. Our purpose is to evaluate whether these stationary models allow one to describe reasonably well the observed ISGs, and thus the average particle drifts all along the tail plasma sheet throughout the inner magnetosphere. It should be stressed here that the very existence of stationary convection in quiet times is currently under debate. The existence of a stationary pattern of particle drift motion averaged from large fluctuations of observed electric field, is called into question (Quinn et al., 1999). The observations of ISG which are possible only in a long-term stationary convection can be used to test this issue.

Four events with well-defined ISGs extended throughout the inner magnetosphere were selected from measurements of the ION spectrometer on board Interball-2. Orbits corresponding to these events cover all MLT sectors in the magnetosphere. The selected events correspond to quiet or moderate levels of disturbance, with the $K_{p}$ index varying from 0 to 3 during the last three days. For these events, model ISGs were calculated using the three convection models mentioned above, assuming the $K_{p}$ index to be equal to zero for two of the four events and equal to one for the other two events. The selected events of ISGs are typical of quiet times. Several other similar events were also analysed and modeled but they will not be shown here.

Besides convection patterns, we also investigated the effect of the particle source location in ISG formation. These particle sources were accounted for in two different ways: 
(a) the "near" particle source corresponding to McIlwain's "injection boundary", as given by Mauk and Meng (1983); Greenspan et al. (1985). This source was defined empirically and, according to McIlwain, it exhibits a burst-like behaviour (i.e. the injection occurs in a transient manner simultaneously along the whole "injection boundary");

(b) the opposite case of a distant source at some distance in the magnetotail (specifically, at and beyond $R_{B}=$ $15 R_{E}$ ).

We anticipate that the use of such different source locations will lead to meaningful variations in model ISGs. It will also allow us to investigate the maximum extent of the "forbidden zones". On the other hand, it is likely that the actual location of the particle source is somewhere between these two extreme cases. To obtain some insights into this location, we traced DMSP-based particle boundaries up to the equatorial plane for all selected cases. The projections of these boundaries were then compared with the "injection boundary" and outer source at $R_{B}=15 R_{E}$. Also, to estimate the location of the "adiabatic" boundary, we calculated the non-adiabaticity parameter, $\kappa$ (Buchner and Zelenyi, 1989), for $15 \mathrm{keV}$ protons using the Tsyganenko (1989) model $\left(K_{p}=0\right)$. As we shall see, it appears from the computation results that the location of the source significantly influences the ISG structure. We will show that ISGs observed from the Interball-2 result from a superposition of both enhanced drift time and "forbidden zones".

\section{Measurements and data}

For comparison of modeling results with observations, it is necessary to select events which occur during relatively quiet times so that the convection can be considered as stationary during sufficiently long time. Indeed, extensive analysis of the ION spectrometer measurements suggests that ISGs are not observed during strongly disturbed conditions at Interball-2 altitude $\left(\sim 3-4 R_{E}\right)$. Two criteria were imposed for data selection: first, the time interval considered has to follow at least two days of weak geomagnetic disturbances. Second, the selected event must cover a wide range of MLT along the orbit. Here is the list of selected events:

(1) 01 January 1997, orbit 519, UT: (4:00-4:25), MLT: (1.6 $\mathrm{h}-2.1 \mathrm{~h})$;

(2) 06 July 1997, orbit 1290, UT: (00:30-2:15), MLT: (6.0 $\mathrm{h}-8.5 \mathrm{~h})$;

(3) 20, 21 May 1997, orbit 1098, UT: (22:00-0:30), MLT: (5.8 h-12.1 h);

(4) 21 November 1997, orbit 1863, UT: (10:00-12:45), MLT: (13.0 h-20.6 h).

The ION experiment on board Interball-2 consists of two ion mass-spectrometers (ION-1, ION-2) and two electron spectrometers (ION-3 and ION-4). All detectors are looking perpendicularly to the satellite spin axis which points toward the Sun. Looking directions of the ION-1 and ION-3 detectors are antiparallel to those of ION-2 and ION-4, so that an angular distribution is measured during a half spin period. ION-1 and ION-2 spectrometers measure $\mathrm{H}^{+}, \mathrm{He}^{++}$, $\mathrm{He}^{+}$and $\mathrm{O}^{+}$fluxes in the energy range $10 \mathrm{eV} / \mathrm{q}-14 \mathrm{keV} / \mathrm{q}$ (for more details, see, for example, Sauvaud et al., 1998a,b).

Figure 1 shows an example of ION data. The spectrograms are for $\mathrm{H}^{+}$. The ion energy is shown in logarithmic scale along the vertical axis, with the intensity of the differential energy flux being coded according to the colour scale on the right. The time variation of the ion pitch angle is also shown below each spectrogram. Clear flux depletions characterised by white spaces with flux intensity below the measurement threshold are noticeable in this figure. We identify these flux depletions as ISGs. One can also see that the energy of ISG increases as the satellite enters the hard radiation belt (note that the apparent break of ISGs inside the radiation belt is an artifact because measurements in this region are strongly corrupted by the noise due to penetrating particles).

Geomagnetic conditions for 19-21 November 1997, 1921 May 1997, 4-6 July 1997 and 30 December 1996 to 01 January 1997 are shown in Figs. 2 and 3. From top to bottom, the following is displayed $K_{p}$ index (data from WDCC2 Centre for Geomagnetism, Kyoto University), $B_{Y}$ GSM, $B_{Z}$ GSM, ion pressure of IMF (WIND data, taken from CDAWeb), $P c$ index (Thule), $A E$ index (data from WDC-C2 Centre). Black horizontal bars indicate the intervals when the modeled ISGs were observed. For all these relatively quiet intervals, we assumed that the magnetospheric electric and magnetic fields were stationary.

\section{Ion gap modeling}

\subsection{Tracing of observed ISGs to the equatorial plane}

To reproduce ISGs observed by Interball-2, tracing was first performed along magnetic field lines into the equatorial plane (more precisely, to the point of minimal $\boldsymbol{B}$-value) using Tsyganenko (1989), assuming $K_{p}=0$. Equatorial $\mathrm{H}^{+}$trajectories were then calculated backward in time. For the sake of simplicity, only particles with $90^{\circ}$ pitch angle at the equator were considered. Though Interball-2 did not perform measurements in the equatorial plane, ISGs' positions may be evaluated there, neglecting the relation between drift velocity and pitch angle. Specifically, the bounce-averaged $\boldsymbol{E} \times \boldsymbol{B}$ drift velocity does not depend on pitch-angle and is such that:

$\left\langle\boldsymbol{V}_{\boldsymbol{E} \times \boldsymbol{B}}\right\rangle=\frac{\boldsymbol{E}_{0} \times \boldsymbol{B}_{0}}{\boldsymbol{B}_{0}^{2}}$,

where $\boldsymbol{E}_{0}$ and $\boldsymbol{B}_{0}$ denote equatorial magnitudes of the electric and magnetic fields. As for the bounce-averaged gradient drift velocity in a dipole field:

$\left\langle\boldsymbol{V}_{\mathrm{grad}}\right\rangle=\frac{6 K}{q B_{0} L} f(\alpha)$, 


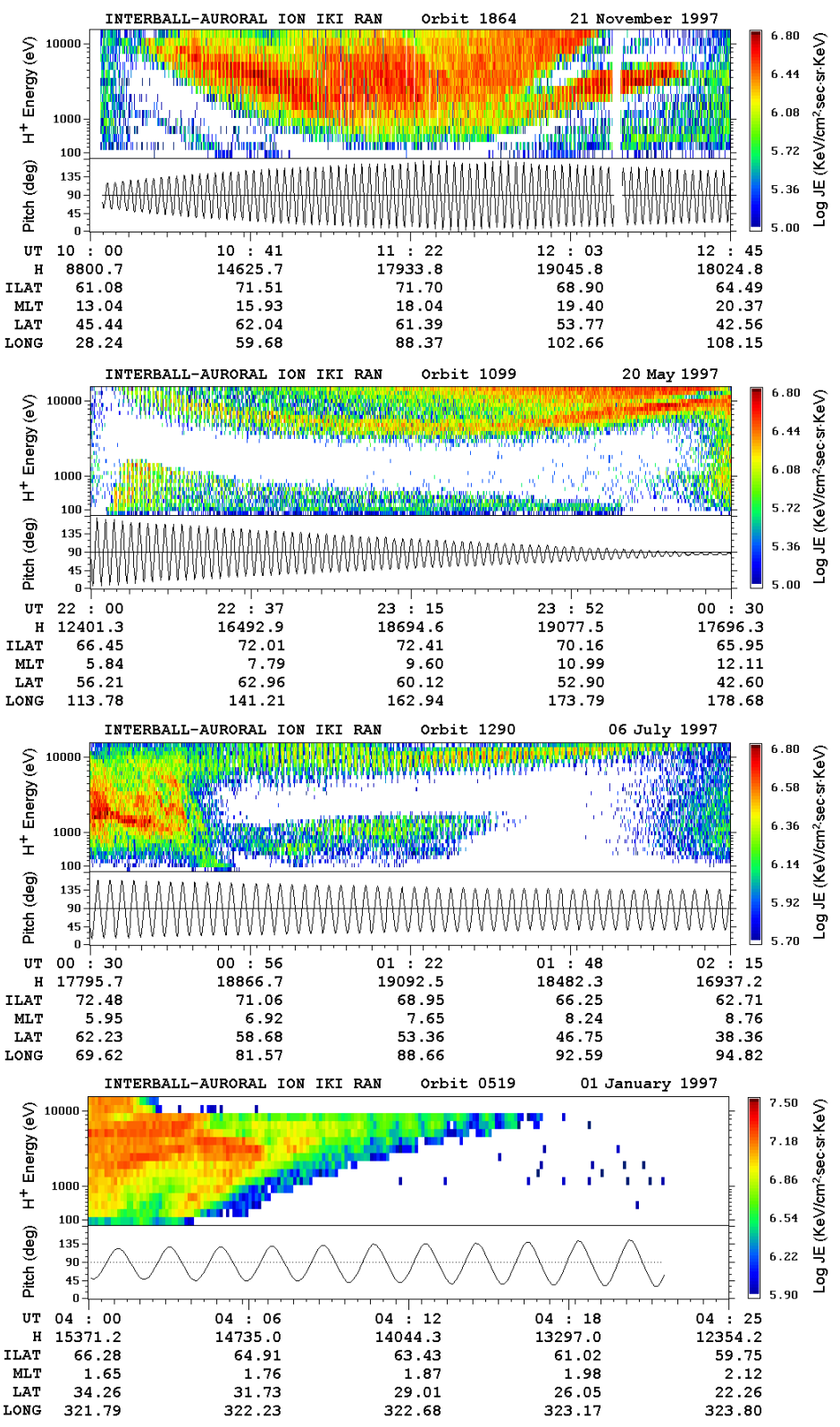

Fig. 1. Proton energy-time spectrograms (from top to bottom) 21 November 1997 (orbit 1864), 20-21 May 1997 (orbit 1099), 6 July 1997 (orbit 1290), 1 January 1997 (orbit 519) with examples, respectively, of ISGs in the evening, dayside, morning and nightside sectors of the inner magnetosphere. At the bottom of each spectrogram the pitch angles of measured protons is shown. Energy flux is colour coded, and the colour-scale is shown on the right side. where $K$ is the kinetic energy of the particles and $f(\alpha) \sim$ $0.35+0.15 \sin \left(\alpha_{0}\right)$ accounts for pitch angle dependence (Roederer, 1970). We assume here that this functional form holds for the quasi-dipole field of the inner magnetosphere. Also, it should be noted that actual particle trajectories may slightly differ from those calculated, but this difference is alleviated by the fact that observed gaps typically cover an energy range $(\Delta E / E) \sim 0.3$. In other words, we focus here on average features of the particle drift paths and not on those achieved at specific energies. Full bounce-averaged drift velocity of particles at some energy is then nearly equivalent to considering the drift velocity of equatorial particles with magnetic moment $K / B_{0}$.

Since we consider particle drifts only in the equatorial plane, it is necessary to map the Interball-2 orbit down to the equator. This mapping was performed with the help of the Tsyganenko (1989) magnetic field model. Invariant radius $R$ and local time MLT were determined at the point of minimum magnetic field. On the other hand, in the simulations, the simple analytical model of Mcllwain (1972) was used for the equatorial magnetic field.

\subsection{Models of particle source}

As mentioned above, the ion source in the magnetotail (assumed constant) was modeled in two different ways, i.e. we considered:

(a) a remote tail source with the inner edge at $R_{B}=15 R_{E}$; 

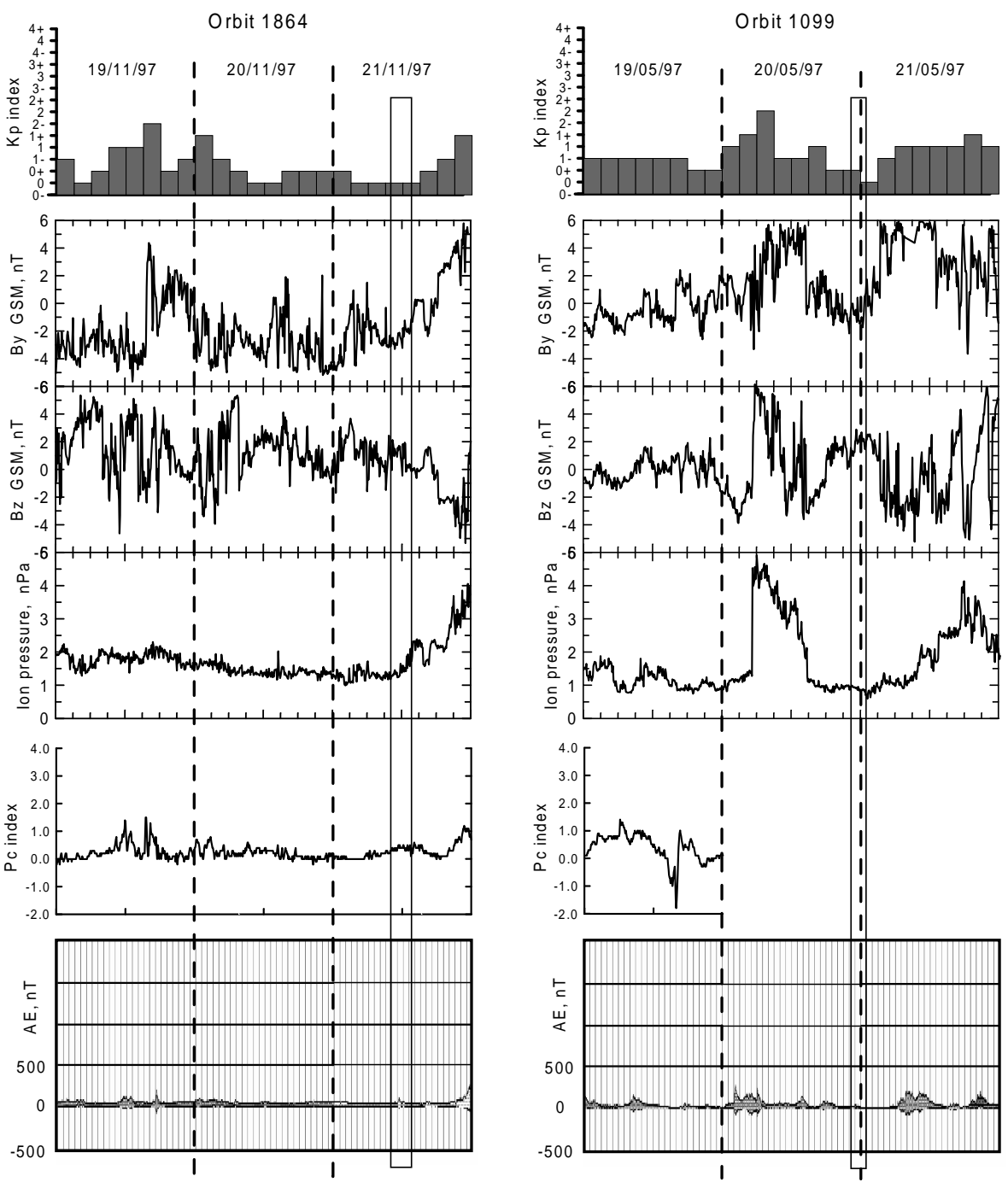

Fig. 2. Geomagnetic conditions for 19 21 November 1997 (left column) and for 19-21 May 1997 (right column). From top to bottom: $K_{p}$ index, $B_{Y}$ GSM, $B_{Z}$ GSM, ion pressure of IMF, $P c$ index, $A E$ index. Black horizontal bars indicate the intervals when the modeled ISGs were observed. (b) McIlwain's "injection boundary" in the form given by Greenspan et al. (1985). This boundary is such that:

$$
R_{B}=\frac{R_{0}^{2}}{\gamma\left(\frac{R_{0}}{\gamma}+\varphi\right)}
$$

in the evening sector (i.e. for MLT ranging from 0 to $-12 \mathrm{~h}$ ) and

$$
R_{B}=\frac{R_{0}^{2}}{\gamma\left(\frac{R_{0}}{\gamma}-\varphi\right)}
$$

in the morning sector (for MLT between 0 and $+12 \mathrm{~h}$ ). One has here: $R_{0}=5.9 R_{E}$ and $\gamma=0.35$. This boundary has the shape of a double spiral and is located much closer to the Earth than the remote tail source in (a).
3.3 Calculation of DMSP boundaries and adiabatic boundary in the equatorial plane

To estimate where the actual particle source is located, we performed tracing of some DMSP-based boundaries into the equatorial plane. This tracing was done with the help of the Tsyganenko (1989) model assuming $K_{p}=0$. For the different periods considered (i.e. 31 December-1 January 1997, 19-20 May 1997, 5-6 July 1997, 20-21 November 1997), the following boundaries were traced:

(a) "b2i" - the points where the ion energy flux above $3 \mathrm{keV}$ has a maximum;

(b) "b2e" - the points where the electron average energy is neither increasing nor decreasing with latitude (one possible interpretation of the edge of the main plasma sheet);

(c) "b3a" - the equatorward most electron acceleration event identified; 

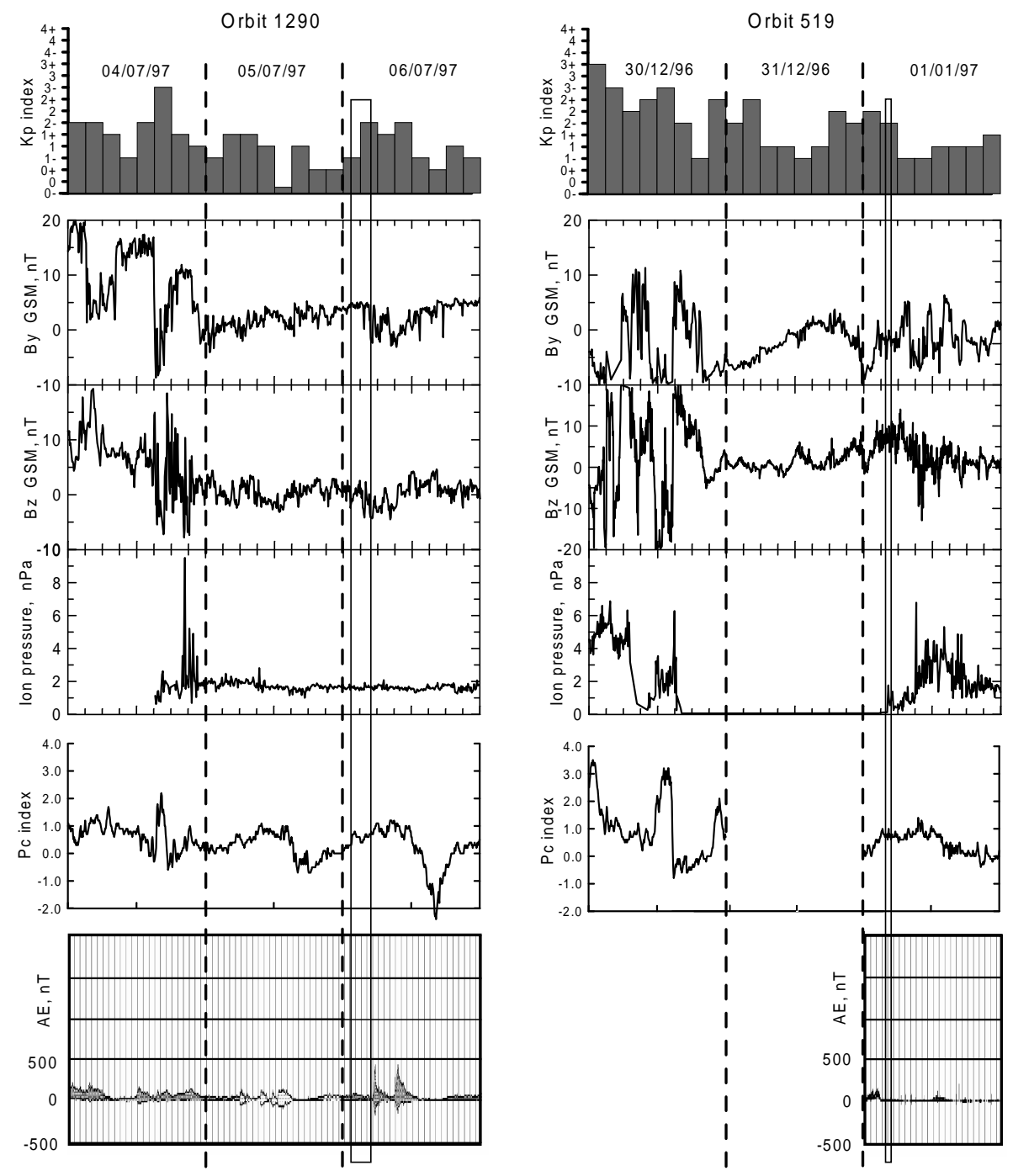

Fig. 3. The same as Fig. 2 for $4-6$ July 1997 (left column) and 30 December 1996 to 01 January 1997 (right column).

(d) "b4s" - the structured/unstructured boundary, based on the running average of correlation coefficients between individual electron spectra and their neighbours.

A more detailed description of these boundaries can be obtained, for instance, in Newell et al. (1996).

The results of these calculations are shown in Figs. 4ad. For comparison, geosynchronous orbit, "injection boundary" and outer source at $15 R_{E}$ are also shown in this figure. It is reasonable to assume that the actual source of ions in the magnetotail is located somewhere between the various boundary projections in the equatorial plane. As a matter of fact, it can be seen in Fig. 4 that most points lie between our model sources. These model ion sources thus appear well justified, with the "injection boundary" providing some "inner" limit for the source location, and $R_{B}=15 R_{E}$ providing some "outer" limit.

Isocontours of the $\kappa$ parameter were also calculated for protons with $15 \mathrm{keV}$ energy, using the Tsyganenko (1989) magnetic field model (assuming $K_{p}=0$ ). At this energy, $\kappa=2$ occurs near $\sim 9 R_{E}$ at midnight. This nonadiabaticity boundary is also shown in Fig. 4. In the magnetic and electric field models adopted, this $\kappa=2$ boundary is located in a region where particles $\boldsymbol{E} \times \boldsymbol{B}$ drift inward rather quickly regardless of energy, so that it may be expected that nonadiabatic motion does not significantly corrupt drift times and leaves ion gaps unaffected.

\subsection{Drift equations for equatorial particles}

By calculating (backward in time) the drift paths and residence times of equatorial particles with energies between $0.1 \mathrm{keV}$ and $15.0 \mathrm{keV}$, we can construct model gaps assuming that particle losses are proportional to the time required to travel from the tail to the spacecraft. Drift velocity equations for equatorial particles in the guiding centre approximation (time-reversed) are:

$$
\begin{aligned}
& \frac{d r}{d t}=\frac{1}{B \cdot r} \cdot\left[\left.\mu \cdot \frac{\partial B}{\partial \varphi}\right|_{r}+\left.\frac{\partial \Phi}{\partial \varphi}\right|_{r}\right] \\
& \frac{d \varphi}{d t}=-\frac{1}{B \cdot r} \cdot\left[\left.\mu \cdot \frac{\partial B}{\partial r}\right|_{\varphi}+\left.\frac{\partial \Phi}{\partial r}\right|_{\varphi}\right]
\end{aligned}
$$




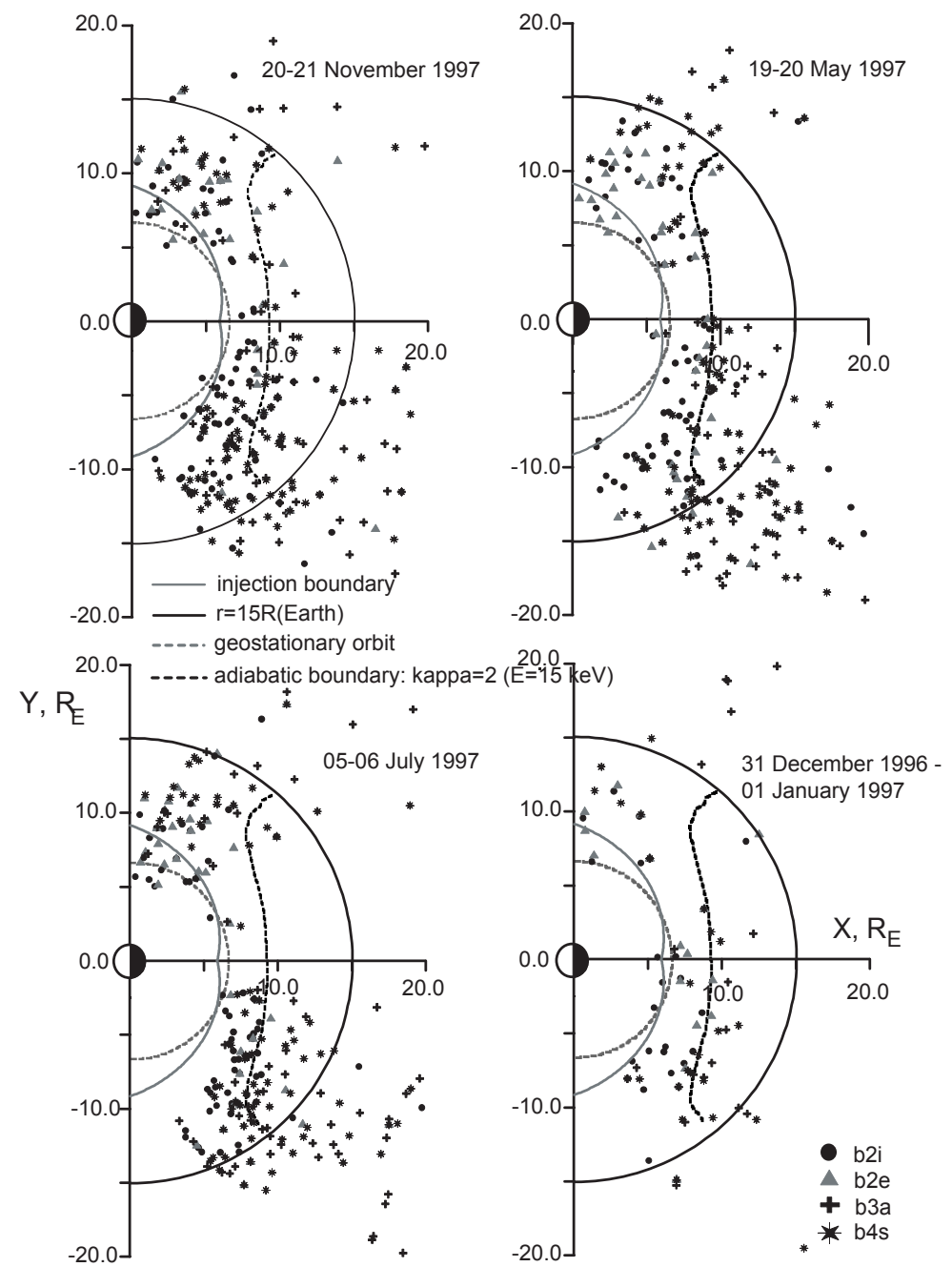

Fig. 4. Location of DMSP particle boundaries in the equatorial plane for the selected events. The "b2i", "b2e", "b3a", "b4s" boundaries are shown (see text). For comparison, the locations of the geostationary orbit, "injection boundary", boundary $R_{B}=15 R_{E}$, and "adiabatic boundary" calculated for the parameter $\kappa=2$, are shown.

Here, $r$ and $\varphi$ are the particle coordinates in the equatorial plane, $\mu$ is the particle magnetic moment divided by particle charge, and $B$ is the magnetic field at the equator. Also, the electric potential is written as:

$\Phi=-\frac{91.5 \mathrm{keV}}{r}+\Phi_{\mathrm{conv}}$,

where $\Phi_{\text {conv }}$ is the model convection potential and the first term describes "corotation", i.e. a transition into non-rotating (magnetospheric) coordinate system (Roederer, 1970).

\subsection{Models of global convection}

Ion gaps were computed using the following convection models:

(1) that of McIlwain (1972) (hereafter referred to as M72), which was constructed from ATS-5 data at geostationary orbit during quiet times. It does not include any dependence on an external parameter, but it is usually regarded as a good description of steady, quiet conditions;
(2) that of McIlwain (1986) (further M86), which explicitly depends on $K_{p}$ and allows for calculations during variable geomagnetic conditions. It differs from the above model especially in the evening and nightside sectors;

(3) that of Volland-Stern (Volland, 1973; Stern, 1975) (further VS75), which also depends on $K_{p}$. It was constructed using only a theoretical approach to the global convection picture, based on average patterns in the polar ionosphere. Its advantage is a simple analytical form that allows for extensive numerical modeling.

In Figs. 5a-c, equipotentials are shown for the three convection models ( $K_{p}$ index is zero). In Fig. 5 d, model isocontours of the magnetic field in the equatorial plane are presented. Dotted lines in Figs. 5a-d schematically show the model magnetopause. The trajectory integration was performed using a Runge-Kutta method (with 4th order of precision). The time step of integration is $0.02 \mathrm{~h}$, and the energy step $0.3 \mathrm{keV}$. Trajectories were traced backward in time up to a model source (either $R_{B}=15 R_{E}$ or McIlwain's "injection boundary"; see introduction above). The time step of the tra- 
(a)

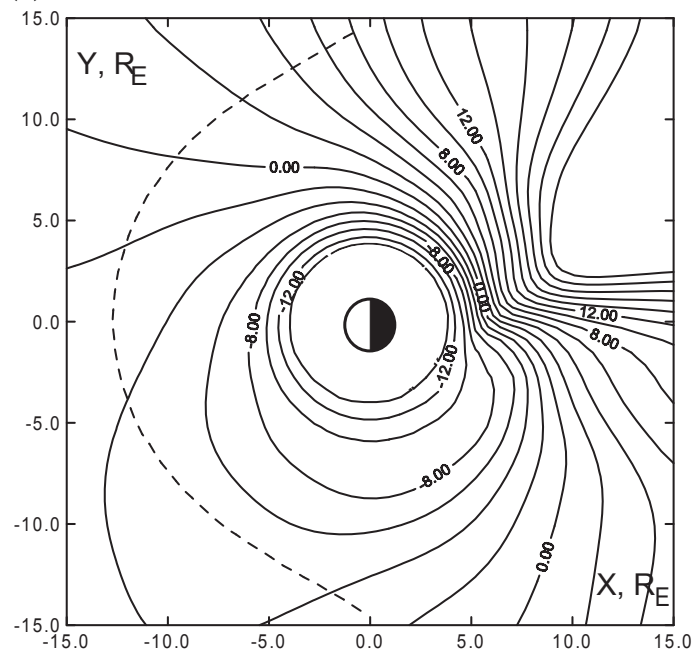

(c)

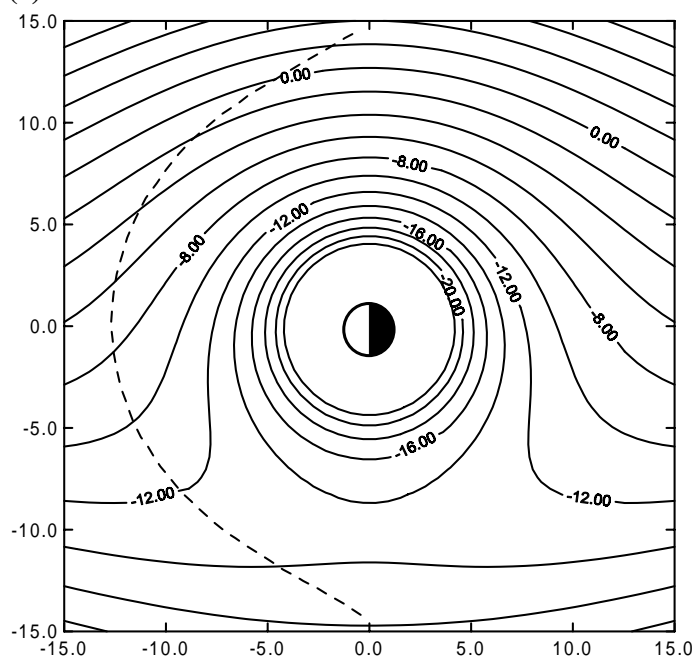

(b)

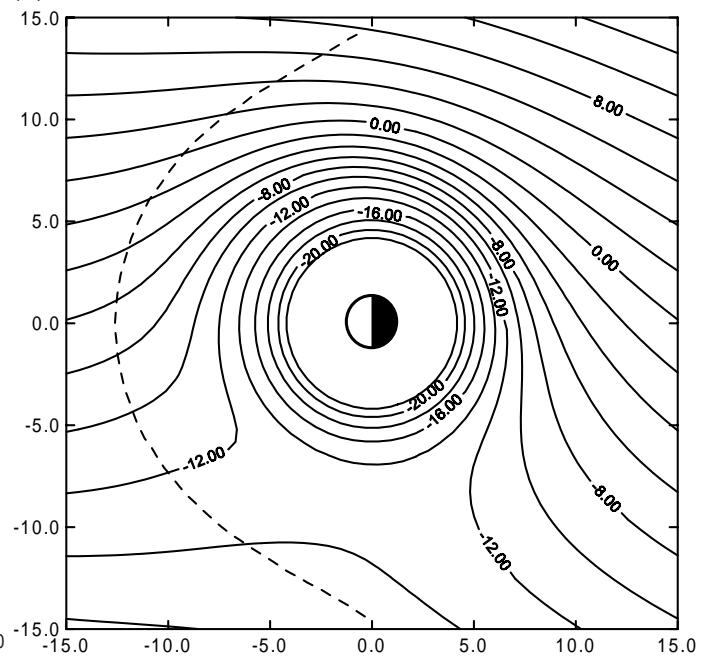

(d)

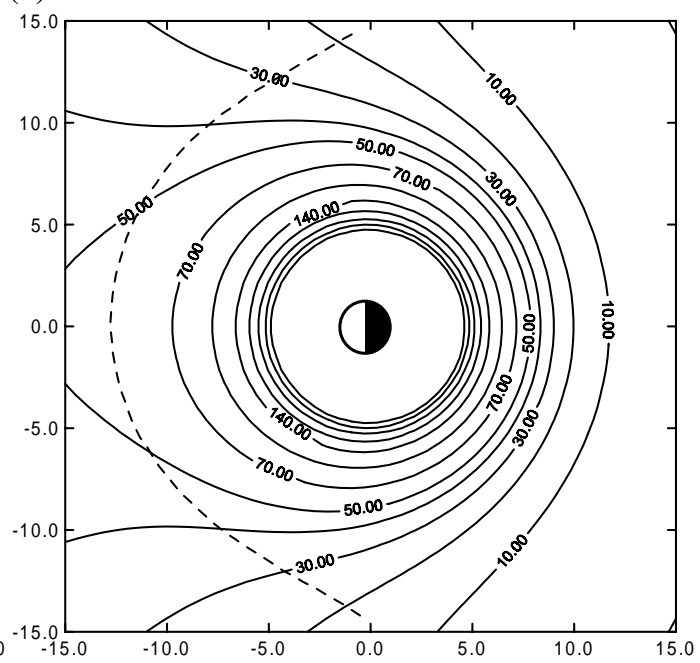

Fig. 5. Electric field equipotentials in the magnetic equatorial plane (in non-rotating magnetospheric frame), according to global stationary convection models by: (a) McIlwain, 1972; (b) McIlwain, 1986; (c) Volland-Stern (Volland 1973; Stern, 1975). (d) contour plot of equatorial magnetic field according to the Mcllwain, 1972 model.

jectory integration along the spacecraft orbit (and along the orbit traced to the equatorial plane) was set to $1 \mathrm{~min}$.

\subsection{Examples of calculated trajectories}

Figure 6 presents examples of trajectories traced backward in time from the Interball-2 location until the remote tail source at $15 R_{E}$ in the magnetotail. Black triangles show the orbit projection in the equatorial plane. Calculations were performed for two different locations along orbits 1864 (viz. 10:30 and 12:00 UT) and 1099 (viz. 22:20 and 23:20 UT). We can see in Fig. 6 that low energy ion trajectories closely coincide with the electric field equipotentials. Note that, if two trajectories with different energies intersect somewhere, a trajectory with any other energy will also pass through this point (Figs. 6a-d and f, g). This feature follows from the fact that we use the adiabatic approximation and time-stationary field models. In Figs. 6a and e, trajectories are calculated for the same initial points but for different convection models (M72 and VS75 in Figs. 6a and e, respectively). It is apparent from these figures that in the case of VS75, trajectories do not encircle the Earth. Ions are rapidly transported into the dayside sector and gaps due to large drift times do not arise. In contrast, in the case of M72, ions with 2-3 keV energy are transported less rapidly, possibly leading to ISGs in energytime spectrograms. Measurements from the ION experiment confirm this outcome, displaying a clear ISG in this energy range (Fig. 1, orbit 1099).

These results demonstrate that modeling of ion gaps critically depends upon the convection pattern adopted. Also, for the case considered in Fig. 6, M72 more accurately reproduces ION measurements than VS75. From Figs. $6 \mathrm{~b}$ and f, it is apparent that trajectories based on M72 and VS75 do not coincide. This suggests that the resulting gaps will also differ widely. In Figs. $6 c$ and d, one can see that in the evening and nightside sectors, there exists a wide range (up to ener- 
M72 convection model (plates a-d)
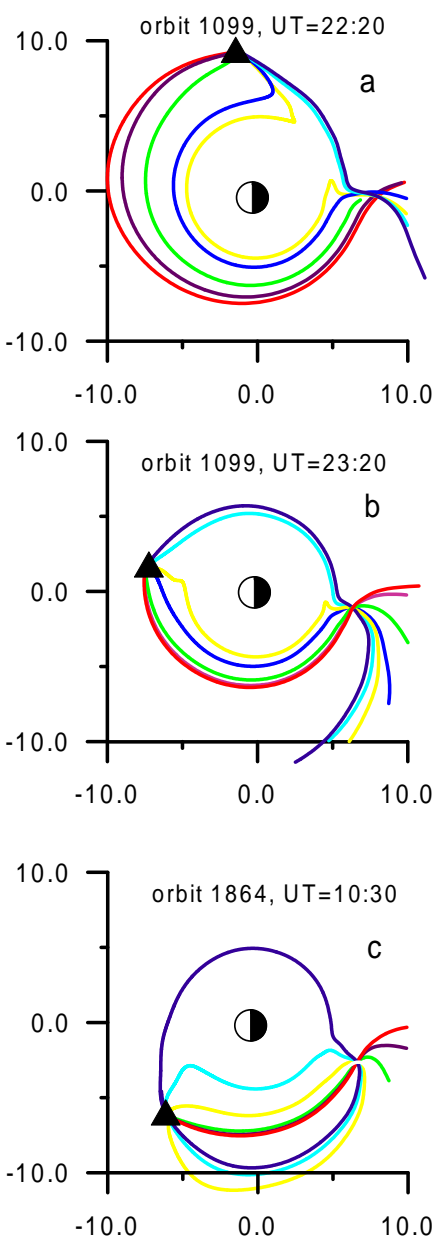

VS75 convection model (plates e-h)
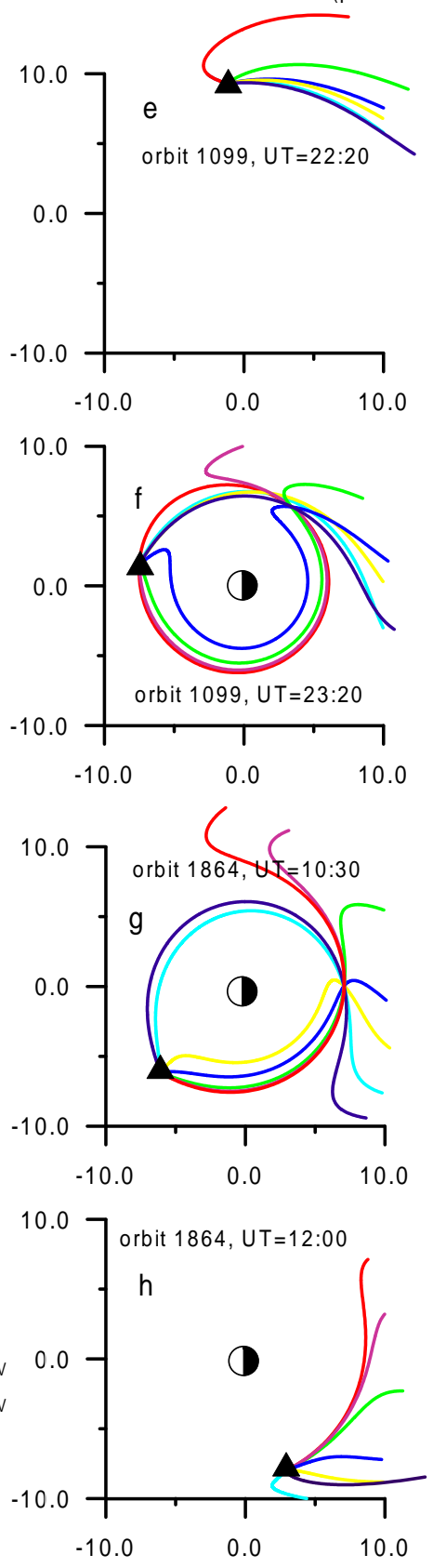

Fig. 6. Examples of the ion trajectories traced back in time from the particular points at the equatorial planeprojections of the Interball-2 orbital points. Black triangles are the points in the equatorial plane. Calculations were made for two points of orbit 1864 (10:30, 12:00 UT) and for two points of orbit 1099 (22:30, 23:30 UT). gies of about $6 \mathrm{keV}$ ) of closed trajectories in the case of M72, whereas for VS75 (Figs. $6 \mathrm{~g}$ and $\mathrm{h}$ ), none of the low energy ion trajectories are closed. Finally, it can be seen in Fig. $6 \mathrm{~h}$ that regardless of energy, particles drift rapidly so that no gap can be obtained.

By analyzing different types of trajectories, one could predict two distinguished types of modeled spectral gaps. The first type is formed by trajectories having enhanced drift time from the model source on the nightside, i.e. mechanism first proposed by McIlwain (1972). The second type with modeled spectral gaps formed by closed trajectories, was pro- posed by Ejiri et al. (1980) and Shirai et al. (1997). For example, particles with energies $>20 \mathrm{keV}$ move along isolines of the magnetic field. As on L-shells $<6$ the magnetic field is near dipole and isolines are resembling circles; the trajectories become closed, so that the model spectral gaps are formed. Particles with low energies move along equipotentials. If the given equipotential line is not connected to the model source, then the trajectories of the corresponding particles will also be closed.

It should be noted that only the drift time calculations were performed, and the particle intensities were not considered. 


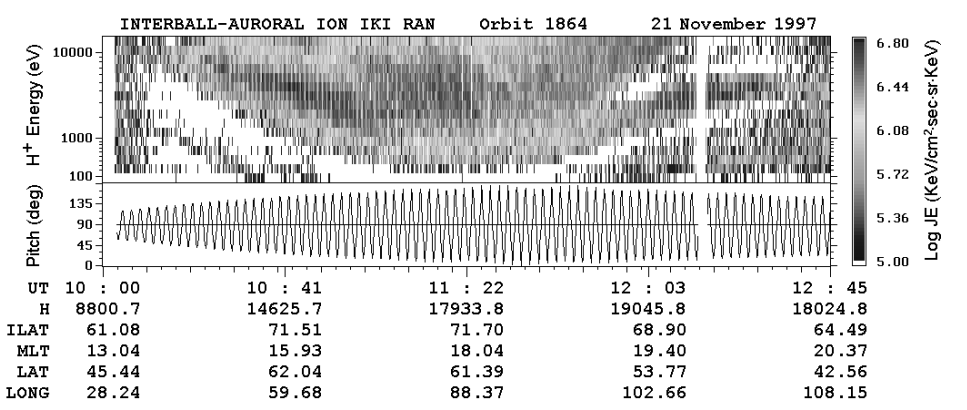

b) convection - Mcllwain,72; model source - inj.boundary
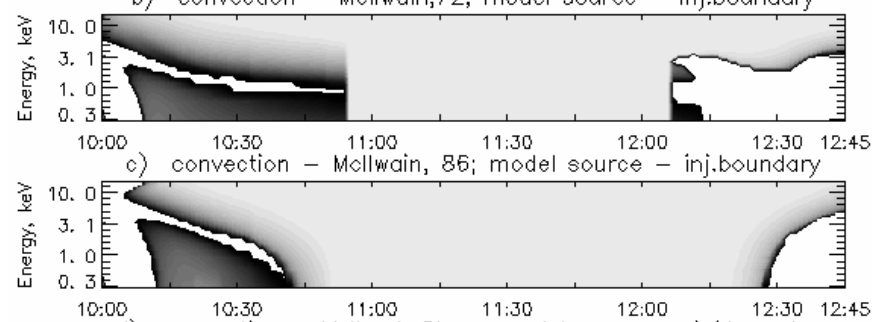

d) convection - Volland-Stern; model source - inj.boundar
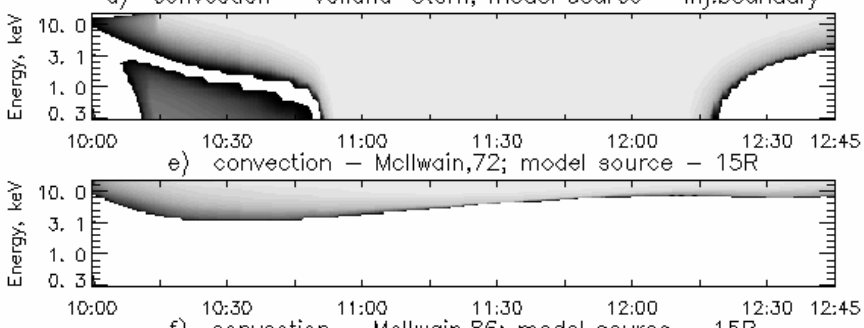

f) convection - Mcllwain,86; model source - 15R

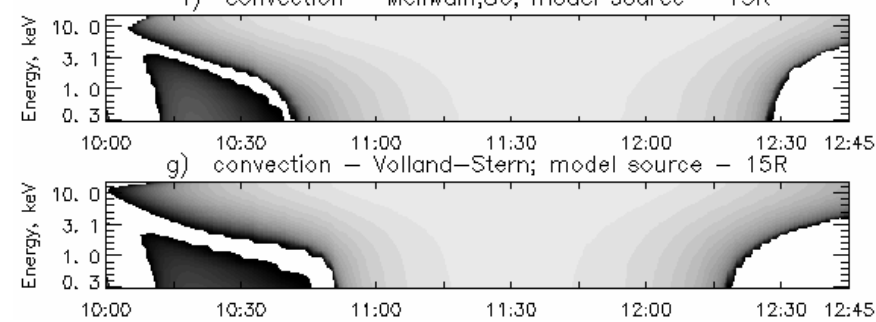

It was also assumed that the magnetic and electric fields are stationary. This approach limits the interpretations of the modeling results: deviations from stationary framework are therefore excluded from our view.

3.7 How to read model spectrograms and compare with ION measurements

Figures 7 to 10 present ION energy-time spectrograms for the four Interball-2 orbits considered (see Sect. 2). Simulation results using the McIlwain "injection boundary" as the ion source in the magnetotail are shown in Figs. 7 to 10b-d. Computations with an outer source at $15 R_{E}$ in the tail are shown in Figs. 7 to $10 \mathrm{e}-\mathrm{g}$. The $X$ and $Y$ axes in these figures give UT and ion energy (in logarithmic scale), respectively, whereas drift times (in hours) are indicated by the grey tones. White bins correspond to ion paths which are disconnected from the source (equivalently, "closed trajectories"; conversely, paths that are connected to the source in the magnetotail will be referred to as "open trajectories" in the fol-
Fig. 7. (a) Proton energy-time spectrograms and pitch angles for 21 November 1997 (orbit 1864). (b)-(g) Modeled spectrograms calculated using the three convection models and the two models of particle source. The calculated drift time is shown by a grey code from zero (light grey) to 40 hours (black). The white spaces correspond to the modeled gaps arising from two different mechanisms - excessive ion drift times and "forbidden zones" for ion coming from the tail. lowing) or which exhibit drift time above $40 \mathrm{~h}$. As mentioned above, in the case of "closed trajectories", we expect an absence of ions in observed energy-time spectrograms, whereas in the case of enhanced drift times, significant attenuation of the ion flux is to be expected. This allows a one-to-one correspondence between white bins in the observed and model spectrograms.

\subsection{Model spectrograms for orbit 1864}

Simulation results for orbit 1864 (MLT range from $13 \mathrm{~h}$ to $20 \mathrm{~h}$ ) are shown in Fig. 7 (panels b-g). A model gap is noticeable in Fig. 7f, using M86 and outer source models. From 10:00 to 10:07 UT, all modeled $\mathrm{H}^{+}$trajectories reaching Interball-2 are closed. Low energy protons drift counterclockwise, approximately along electric field equipotentials. On the other hand, high energy protons drift clockwise, approximately along isolines of the magnetic field, whereas protons at intermediate energies (with energies $\sim 2-7 \mathrm{keV}$ ) drift into the evening sector along closed trajectories and 


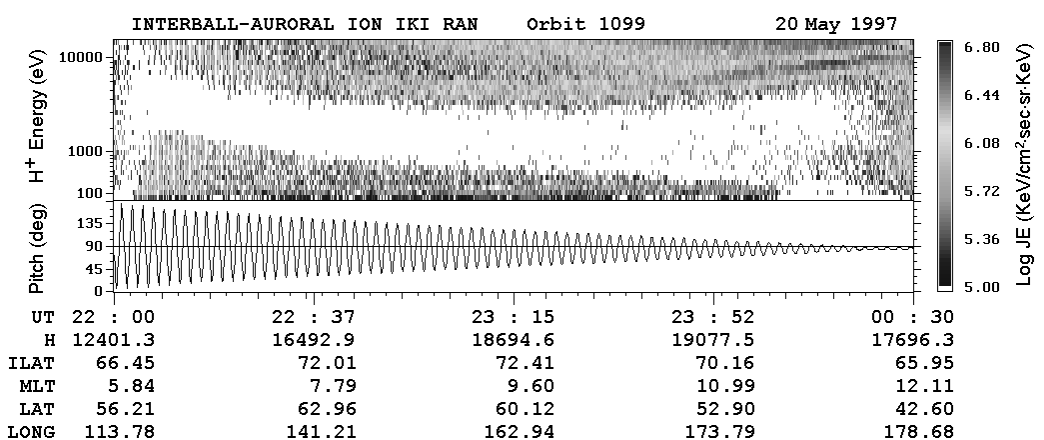

b) convection - Mcllwain,72; model source - inj.boundary
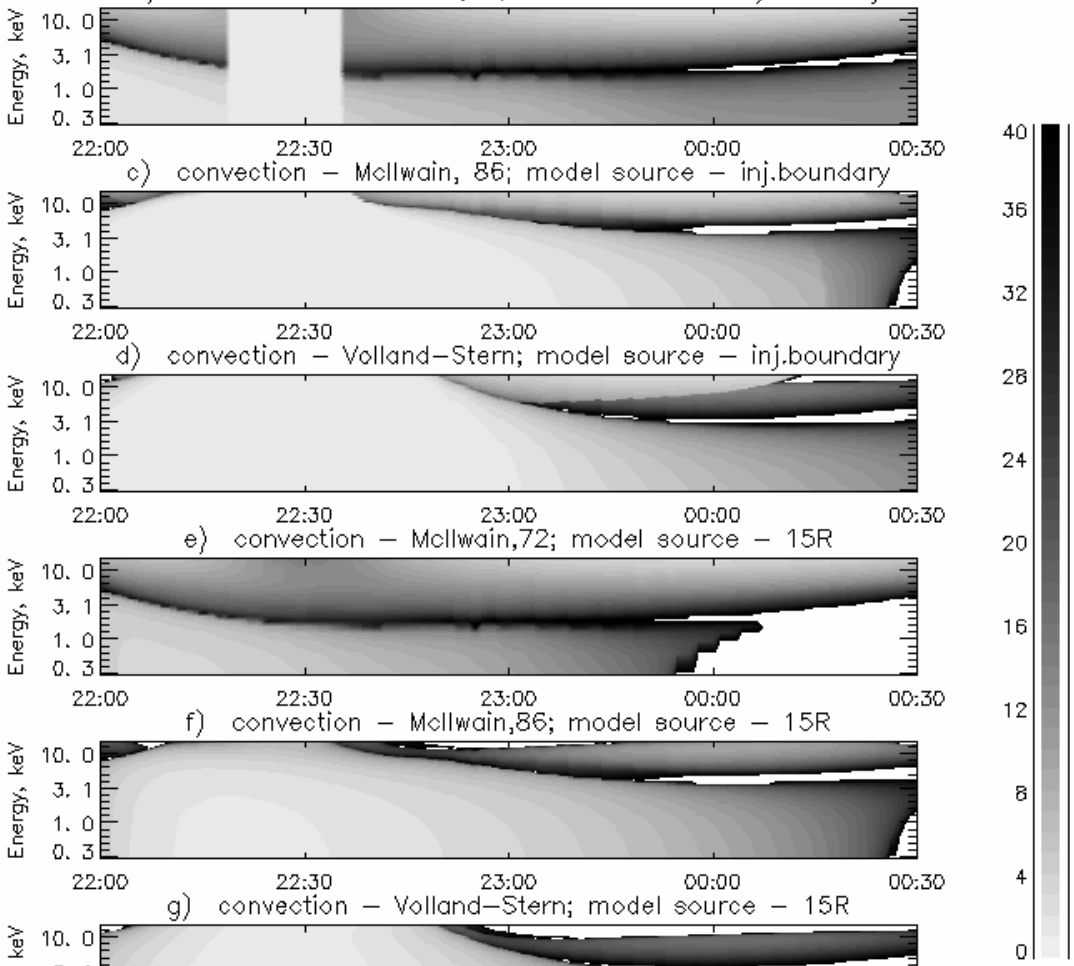

e) convection - Mcllwain,72; model source - 15R

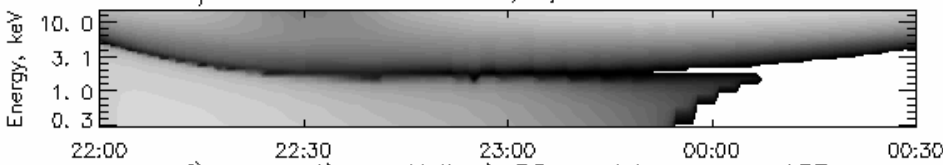

f) convection - Mcllwain,86; model source - 15R

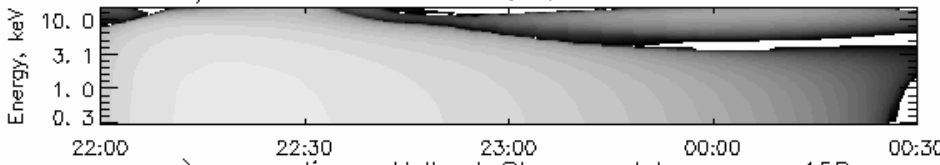

g) convection - Volland-Stern; model source - 15R

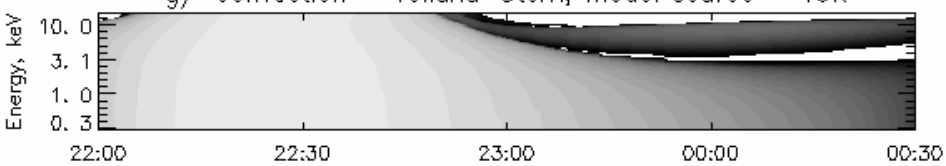

Fig. 8. Same as Fig. 7 for 20-21 May 1997 (orbit 1099).

do not encircle the Earth. From 10:07 UT, Interball-2 enters into the region, where for given convection model trajectories, particles with energies $\sim 5-8 \mathrm{keV}$ become open, until 10:10UT, where there are closed particle trajectories. At medium energies, a region of "resonant" behaviours appears, characterised by drift times between the source and the spacecraft up to $40 \mathrm{~h}$. This leads to a clear ISG. Until 10:30 UT, no closed trajectory can be found and enhanced drift times at medium energy $(3 \mathrm{keV}$ for $10: 15 \mathrm{UT} ; 1.5 \mathrm{keV}$ for 10:30 UT) persist. Finally, from 12:25 UT, closed trajectories at low energies reappear.

Quite a different pattern is obtained if M72 is used (Fig. 7e), i.e. instead of a long and wide strip of enhanced drift times, Fig. 7e exhibits a region of closed trajectories that is inconsistent with Interball-2 measurements. Still, a gap due to large drift times may be achieved if one uses the "injection boundary" for the ion source (Fig. 7b). This result follows from the fact that the "injection boundary" is located closer to Earth than $15 R_{E}$. Since closed equipotentials do not reach $15 R_{E}$ in $\mathrm{M} 72$, closed trajectories at low energies arise, leading to a pronounced gap. In contrast, the "injection boundary" intercepts M72 closed equipotentials so that trajectories originating from this boundary are not considered as forbidden. Gaps due to large drift times may still occur though, and this comparison clearly illustrates the crucial role of source location in ion gap formation.

Finally, Figs. 7b-d show the gaps obtained along orbit 1864 considering all three convection models and the "injection boundary" as the ion source. For M86 and VS75, clear changes in the gap structure are noticeable, namely the shape of the forbidden region is different and the gap energy range is lower than in the case of $15 R_{E}$. From a qualitative 


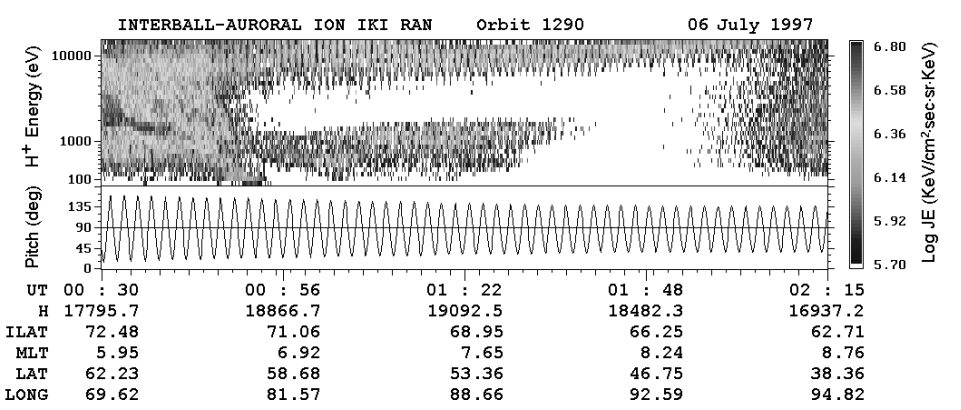

b) convection - Mcllwain,72; model source - inj.boundary
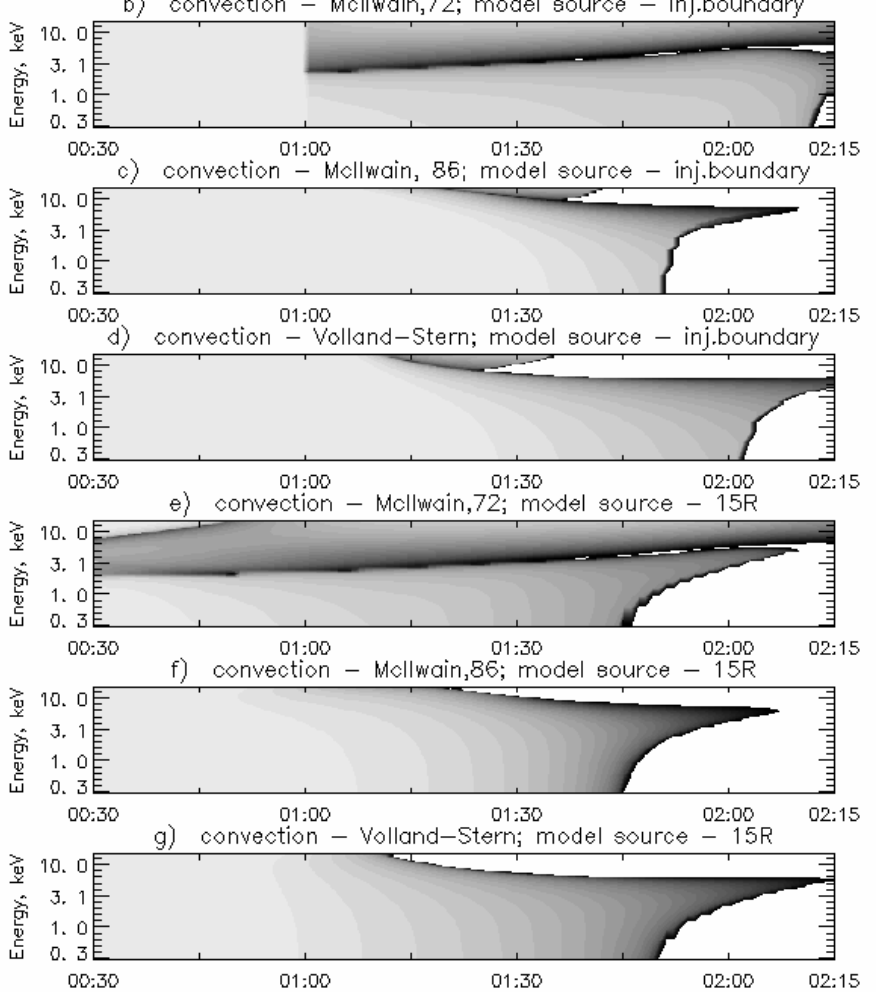

viewpoint, the overall gap patterns are similar, exhibiting a combination of enhanced drift times and forbidden trajectories.

\subsection{Comparison with ION measurements}

In Fig. 7a, the large gap observed in ION measurements is consistent with that obtained numerically as a result of extremely large drift times (up to $\sim$ 11:00 UT on modeled spectrograms). Also, ISGs at low energies from 10:07 UT to 10:20 UT in Fig. 7a seemingly correspond to regions of closed trajectories. Figures 8 to 10 (panels b-g) show model gaps for other Interball-2 events. Here again, the gap development is due to superposition of two effects (large drift times and closed trajectories). As for the ion source, the main difference between "injection boundary" and outer source at $15 R_{E}$ is that trajectories that are viewed as closed in the latter case are open in the former case (see Sect. 3.8). This leads to quite distinct forbidden domains.

Besides the influence of the source location on modeled
Fig. 9. Same as Fig. 7 for 6 July 1997 (orbit 1290).

ISGs, there are also a number of reasons for the discrepancies between modeled and actually observed spectrograms:

(1) The considered convection models do not work properly in some sectors of the magnetosphere. For example, in the late evening sector (Fig. 7, orbit 1864), none of the convection models can reproduce observed ISGs from 11:30 UT to $12: 30 \mathrm{UT}$;

(2) The calculations made show only drift times, not intensities of drifting particles. We do not consider the ions previously trapped on closed trajectories. The existence of these ions leads to "painting" of white forbidden zones, and modification of the observed stationary picture;

(3) We do not consider other possible sources of particles. For example, ionospheric sources can also supply ions to the closed trajectories;

(4) We assume that model particle source is distributed all along the boundary, and it is stationary in time. If we as- 


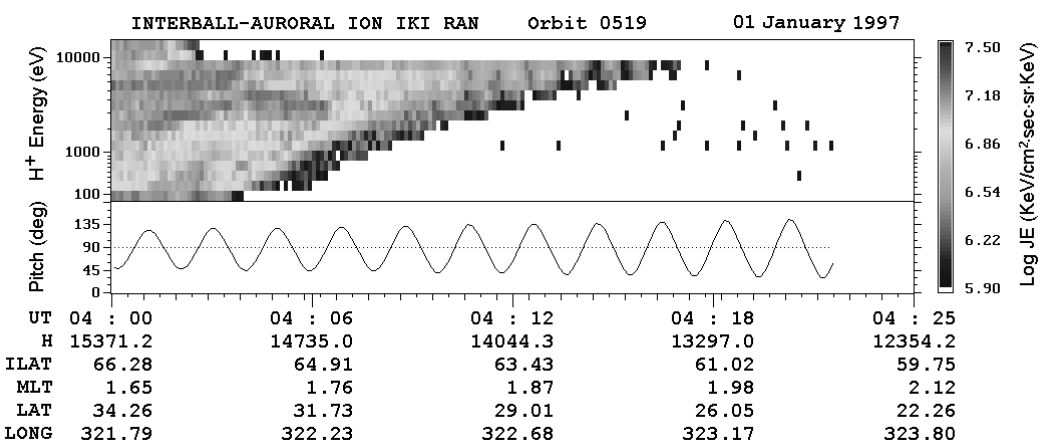

b) convection - Mcllwain, 72; model source - inj.boundary

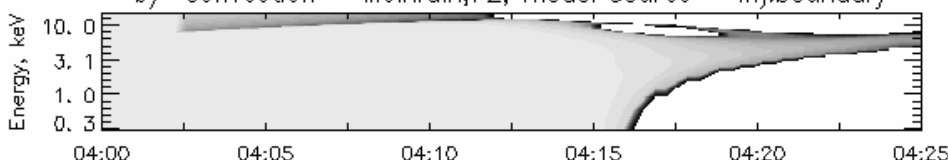

c) convection - Mollwain, 85; model source - inj.boundary
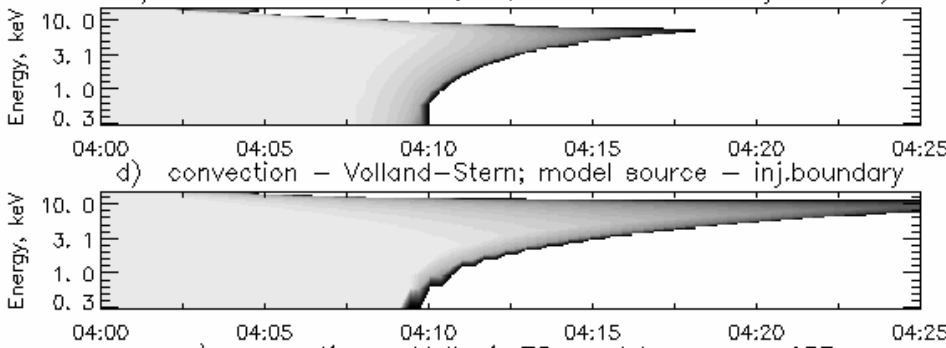

e) convection - Mcllwain,72; model source - 15R
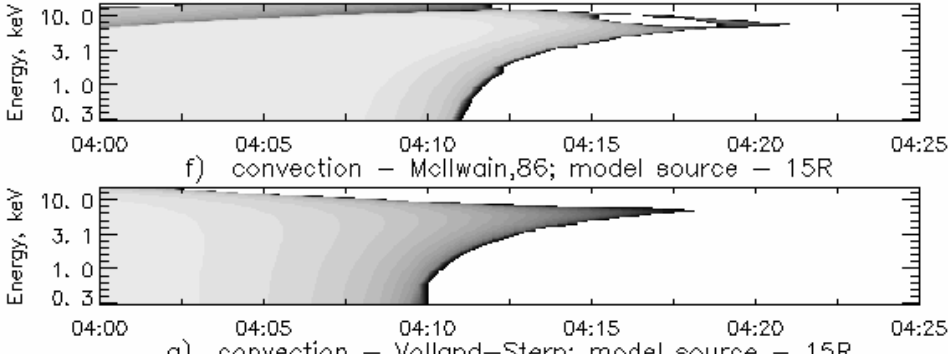

g) convection - Volland-Stern; model source - 15R

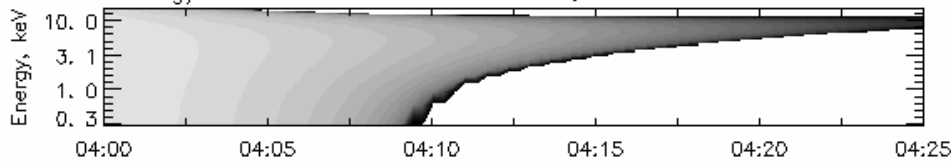

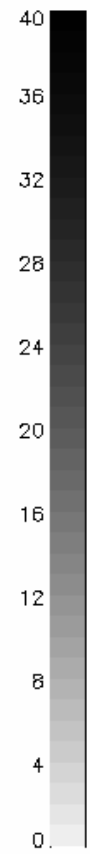

Fig. 10. Same as Fig. 7 for 1 January 1997 (orbit 519). sume that model source is confined to some MLT range, it would lead to additional gaps on model spectrograms. The burst-like behaviour of particle source can also deviate ISGs' pattern from a stationary position. Using deviations of real particle source from a stationary position and that are uniform all along the boundary, one can explain the discrepancies between modeled and observed gaps for orbit 1099 (Fig. 8).

\section{Discussion}

Three different models of the large-scale magnetospheric convection were considered. First of all, the numerical results show that there is a qualitative agreement between modeled and observed ISGs for the four selected orbits when
M72 is used (with the exception of the late evening sector, where all three convection models do not reproduce the observed ISGs' pattern). For the other two models, the agreement is not so good in the morning (orbit 1290), in the morning-dayside sectors (orbit 1099), and in the late evening sector (orbit 1864). In the dayside and early evening sectors, observed ISGs are reasonably well described by all three models. In the sector extending from $\sim 20$ MLT and later (Figs. $7 \mathrm{~b}$ and e), the agreement using M72 is rather poor. This agreement may be improved though if the source is placed farther than the "injection boundary" but nearer than $15 R_{E}$. This supports the conclusion that the actual particle source is located somewhere between these two boundaries. For the other two models in the early morning and late evening sectors, all ions are rapidly transported away from 
the source and there is no mechanism leading to gap formation in the present modeling scheme. It is clear from the above results that ISGs observed by the ION spectrometer may be divided in two types: those due to forbidden trajectories and those due to increased drift times. Let us consider these in more details.

4.1 Modeling of gaps in the nightside, evening sectors and the first type of gaps - a "nose" structure

The first type of ISG is usually observed at low L-shells near the equatorial boundary of the diffuse auroral zone, when the spacecraft enters the hard radiation region of the outer radiation belt. According to the contemporary views, this region is close to the large-scale convection boundary, or the plasmapause. Such features were sometimes described as "nose" structures observed during quiet or weakly disturbed times. They have been reported and successfully modeled in various studies (Ejiri et al., 1980; Shirai et al., 1997). It needs to be noted that there exists another type of the "nose" structures which appear during and after strong injections in disturbed times (see, for example, Ganushkina et al., 2000), but they are not relevant to steady conditions considered here. In the Interball-2 measurements, numerous examples of quasisteady "nose" ion gaps can be seen in early morning and nightside passes. A striking example of such a "nose" structure is shown in Fig. 10a. As discussed above, the gap displayed here can be explained as the result of open/closed ion trajectories. It is clear from Figs. 10b-g that regardless of the convection model used, the computed and observed forbidden regions are similar.

By comparing the modeled and observed ISGs, it is possible to draw some conclusions about the convection electric field in the inner magnetosphere. In Fig. 10a, the observed low energy cutoff takes place near 04:07 UT, while that obtained from the computations occurs near 04:10 UT. Since this cutoff coincides with the crossing of the first closed electric field equipotential (particles with "zero" energy only experience $\boldsymbol{E} \times \boldsymbol{B}$ drift and move along these equipotentials), we may infer that the actual corotation area was extending toward higher L-shells than in the numerical models.

As mentioned above, "nose" structures are observed by the ION spectrometer in the near-midnight sector of the magnetosphere, within the $\sim 20-02 \mathrm{~h}$ MLT range. In all the cases considered, they may be explained in the same manner, namely the existence of open and closed trajectories. The "nose" energy (i.e. the energy of particles penetrating to the lowest L-shells) is $\sim 10 \mathrm{keV}$ both in the observations and in the computations. Shirai et al. (1997) performed calculations of open/closed trajectories for different values of the convection field strength (equivalently, for different values of the $K_{p}$ index). Not unexpectedly, these calculations indicate that when the electric field magnitude increases, particles penetrate deeper toward the Earth and the "nose" energy increases.
4.2 The other type of gaps - ISGs due to maximum drift time

In the morning, dayside and evening sectors, ISGs of the second type (i.e. due to enhanced drift times) appear in addition to "nose" structures. In the ION measurements, three ISGs at different energies can thus be identified at the same time, separated by distinct borders. In the dayside sector (orbit 1864, 10:00-11:00 UT, Fig. 7; and orbit 1099, 23:4500:30 UT, Fig. 8), the observed gaps may be adequately described by all three convection models. In M72, an ion gap arises due to increased drift time when the "injection boundary" is taken as the source. In contrast, this gap vanishes if injection is displaced to the outer source at $15 R_{E}$. Here, the corotation regime extends over a wider domain that reaches the magnetopause in the evening sector (see also Fig. 5). At a given energy, the region of forbidden trajectories expands accordingly in the evening sector. As a result, low energy protons launched from a distant source in the nightside magnetosphere cannot penetrate into the dayside sector. On the other hand, the same M72 model combined with the "injection boundary" source leads to large drift time gaps. These two mechanisms of gap formation lead to qualitatively different ISGs, i.e. low-energy cutoff for the former and narrow "resonant" energy range for the latter.

In the morning and dayside sectors (MLT from $6 \mathrm{~h}$ to $12 \mathrm{~h}$ ) of orbits 1099 and 1290, observed ISGs are well described only within the M72 convection model. These observed ISGs in the $\sim 3-8 \mathrm{keV}$ energy range are in agreement with model gaps due to large drift times. Gaps resulting from forbidden zones are also observed, responsible for ion cutoff at low energies. These latter gaps are well described within M72 as well. As for VS75 and M86, these models yield large drift time gaps, but there is a poor agreement between their overall structure and that observed. It is also interesting that in the early morning sector (MLT 4-5 h), VS75 does not lead to large drift times but to a "nose" structure (Fig. 9g). This "nose" energy is comparable to that of the M72 gap (Figs. 9e and $\mathrm{g}$ ).

Shirai et al. (1997) proposed that at MLT $\sim 6 \mathrm{~h}$, ISGs can be formed only from "forbidden zones". They used the VS75 model in calculation, and the obtained model ISGs were similar to the ones shown in the Fig. 9g. To explain the existence of particles at high energies, they took into account previously trapped particles. But from Figs. 9e and g, one could derive two alternative explanations for the ISGs in the early morning sector.

The first way, proposed by Shirai et al., is illustrated by Fig. 9g. If we suppose the presence of a population of trapped particles at energies $12-15 \mathrm{keV}$, then the obtained modeled picture would be slightly similar to the observed.

The second way is that observed ISGs are due to enhanced drift time, as shown in Fig. 9e. Here, in order to obtain good agreement with the observations, there is no reason to consider previously trapped particles. 


\subsection{Particle source location}

Despite the above described limitations, modeling of ion gaps also helps to draw conclusions regarding the location of the particle source in the magnetotail. For the evening sector, if the "source" is far from the Earth, low energy particles do not penetrate deep inside the inner magnetosphere because the quiet time electric field is weak and the region of closed equipotentials is consequently wide. Therefore, for a gap to develop as a result of large drift times, particle injection should occur not further than some limiting distance which may be directly evaluated through numerical modeling. On the other hand, it is apparent from Figs. $8 \mathrm{~b}$ and e that an outer source at and beyond $15 R_{E}$ is more consistent with the observations. In Fig. 8b, resorption of the gap from $22: 18$ UT to $22: 37$ UT is due to the fact that Interball-2 is located beyond the model "injection boundary". In this case, agreement between the computed and model ISGs requires a source located relatively far enough from the Earth, typically tailward of $8 R_{E}$.

In summary, for orbit 1864, the "injection boundary" yields a better fit to the data, whereas an outer source at $15 R_{E}$ seems more appropriate for orbit 1099 . The cause of this difference is that the "injection boundary" model does not account for a particular physical mechanism of particle injection. Rather, it provides an empirical description of the inner limit of plasma injections (Lopez et al., 1990). In addition, these injections were initially viewed as short-term bursts as opposed to the more or less continuous flow which may be expected during quiet times. Thus, in one case, injection may occur far from the Earth, which justifies the use of an outer source at $15 R_{E}$, whereas in the other case, injection occurs closer to the Earth and the "injection boundary" is appropriate. Then, the definition of "quiet times" obviously needs to include the possibility of weak substorm-like injections at high latitudes. It can be concluded from the present study that ISGs allow one to verify the existence of quasi-steady convection in the inner magnetosphere during quiet times. Quasi-steadiness here refers to the "average" (in a spatially extended area and over a long time period), large-scale convection and not to the nearly instantaneous local measurements. Nowadays, such information is hardly attainable via other methods. Given the observed structure of the ISGs, one could, in principle, infer the global convection pattern over broad areas during the preceding hours.

\section{Conclusions}

(1) There is a good qualitative agreement between ISGs observed by the spectrometer ION on board the Interball2 at altitudes $\sim 10000-20000 \mathrm{~km}$ in all MLT sectors through the inner magnetosphere during quiet times and modeled gaps. The results of modeling are sensitive to the assumed global stationary convection model, especially in the early morning and late evening sectors of the inner magnetosphere. The best agreement is found for the McIlwain, 1972 convection model.

(2) The modeling results are significantly influenced by the assumed particle source location. The data studied are all consistent with the source location in the near tail at distances ranging from the "injection boundary" location until $10-15 R_{E}$. For particular cases, this approach makes it possible to approximately estimate the real source location in the nightside plasma sheet from the modeling of ISGs.

(3) The observed ISGs are shown to be the results of the superposition of two different factors: first, the gaps due to excessive drift time for particular "resonant energy" ions from the source to the observation point, and second, the gaps due to the existence of "forbidden" zones disconnected from the source in a particular energy range. Both factors were described in the literature, but considered separately, while the observed global pattern actually includes both of them but in particular MLT sectors.

(4) For the formation of ISGs, a quiet period with a duration of tens of hours is necessary to achieve quasi-stationary particle drift motion (i.e. for the drift times of low energy ions throughout the inner magnetosphere).

(5) The ISG observations directly verifies the existence of a global stationary average convection pattern during quiet times, despite the evidence of large amplitude local electric field fluctuations.

Acknowledgements. WIND data taken from CDAWeb are courtesy of R.Lepping and K.Ogilvie. Authors are indebted to P.Newell who provided us with the DMSP data. The work was supported by the NASA JURRISS Grant NAG5-8638 for NYB and YIG, by INTAS Grant 2000-465, by French-Russian cooperation program (PICS and RFBR) Grant 00-02-22001, and by joint RussianAustrian RFBR Grant 01-02-02005 for NYB.

Topical Editor G. Chanteur thanks two referees for their help in evaluating this paper.

\section{References}

Buchner, J. and Zelenyi, L. M.: Regular and chaotic charged particle motion in magnetotaillike field reversal, 1, basic theory of trapped motion, J. Geophys. Res., 94, 11 821, 1989.

DeForest, S. E. and McIlwain, C. E.: Plasma clouds in the magnetosphere, J. Geophys. Res., 76, 3587, 1971.

Delcourt, D. C., Belmont, G., Sauvaud, J.-A., Moore, T. E., and Martin Jr., R.: Centrifugally driven phase bunching and related current sheet structure in the near-Earth magnetotail, J. Geophys. Res., 101, 19839, 1996.

Ejiri, M., Hoffman, R., and Smith, P.: Energetic particle penetrations into the inner magnetosphere, J. Geophys. Res., 85, 653 1980.

Ganushkina, N. Y., Pulkkinen, T. I., Sergeev, V. A., Kubyshkina, M. V., Baker, D. N., Turner, N. E., Grande, M., Kellett, B., Fennell, J., Roeder, J., Sauvaud, J.-A., and Fritz, T. A.: Entry of 
plasma sheet particles into the inner magnetosphere as observed by Polar/CAMMICE, J. Geophys. Res., 105, 25 205, 2000.

Greenspan, M., Williams, D., Mauk, B., and Meng, C.-I.: Ion and electron energy dispersion features detected by ISEE-1, J. Geophys. Res., 90, 4079, 1985.

Kovrazhkin, R., Sauvaud, J.-A., and Delcourt, D.: Interball-Auroral observations of $0.1-12 \mathrm{kev}$ ion gaps in the diffuse auroral zone, Ann. Geophysicae, 17, 734, 1999.

Li, X., Baker, D. N., Temerin, M., Reeves, G. D., and Belian, R. D.: Simulation of dispersionless injections and drift echoes of energetic electrons associated with substorms, J. Geophys. Res., 25, 3763, 1998.

Lopez, R., Sibeck, D., McEntire, R., and Krimigis, S.: The energetic ion substorm injection boundary, J. Geophys. Res., 95, 109, 1990.

Mauk, B. and Meng, C.-I.: Characterization of the geostationary particle signatures based on the "injectioin bounary" model, J. Geophys. Res., 88, 3055, 1983.

McIlwain, C.: Plasma convection in the vicinity of the geosynchronous orbit, in: Earth's Magnetospheric Processes, (Ed) McCormac, B., p. 268, D. Reidel Pub.Comp., 1972.

McIlwain, C.: A Kp-dependent equatorial electric field model, Adv. Space Res., 6, 187, 1986.

Moore, T., Arnoldy, R., Feynmann, J., and Hardy, D.: Propagating substorm injection fronts, J. Geophys. Res., 86, 6713, 1981.

Newell, P., Feldstein, Y., Galperin, Y., and Meng, C.-I.: The morphology of nightside precipitation, J. Geophys. Res., 101,
$10737,1996$.

Quinn, J. M., Paschmann, G., Sckopke, N., et al.: EDI convection measurements at 5-6 $R_{E}$ in the post-midnight region, Ann. Geophysicae, 17, 1503, 1999.

Roederer, J.: Dynamics of Geomagnetically Trapped Radiation, in: Pysics and Chemistry in Space, v.2, Springer-Verlag, New York, 1970.

Sauvaud, J.-A., Barthe, H., Aoustin, C., Thocaven, J., Rouzaud, J., Penou, E., Kovrazhkin, R., Afanasiev, K., and Ivanchenkova, I.: Measurement of the suprathermal plasma by ION spectrometric complex on the Interball-2 satellite (Auroral probe), Cosmic Research, 36, 59, 1998a.

Sauvaud, J.-A., Barthe, H., Aoustin, C., Thocaven, J., Rouzaud, J., Penou, E., Popescu, D., Kovrazhkin, R., and Afanasiev, K., The ION experiment onboard the Interball-Auroral satellite; initial results on velocity-dispersed structures in the cleft and inside the auroral oval, Ann. Geophysicae, 16, 1056, 1998 b.

Shirai, H., Maezawa, K., Fujimoto, M., Mukai, T., Saito, Y., and Kaya, N.: Monoenergetic ion drop-off in the inner magnetosphere, J. Geophys. Res., 102, $19873,1997$.

Stern, D.: The motion of a proton in the equatorial magnetosphere, J. Geophys. Res., 80, 595, 1975.

Tsyganenko, N.: A magnetospheric magnetic field model with a warped tail current sheet, Planet. Space Sci., 37, 5, 1989.

Volland, H.: A semiempirical model of the large-scale magnetospheric electric fields, J. Geophys. Res., 78, 171, 1973. 